\title{
Escleroterapia em lesões vasculares de boca: relato de dois casos clínicos
}

\author{
Sclerotherapy in vascular lesions of the mouth: report of two \\ clinical cases
}

\author{
Erick Alves Pereira* \\ Tiago França Araripe Cariri*
}

\section{Resumo}

O hemangioma é uma neoplasia de comportamento benigno, determinada pela proliferação anormal de vasos sanguíneos. São lesões assintomáticas, porém, seu crescimento progressivo pode acarretar danos traumáticos locais, ocasionando dor, ulcerações e até sangramentos, que, dependendo da localização, se tornam de difícil controle, principalmente quando de natureza arterial. Com considerável incidência na região de cabeça e pescoço e na cavidade bucal, acomete mais frequentemente a região de mucosa jugal, lábios e língua. Tendo em vista a sua relevância clínica, por estar presente na sua área de atuação e ser relativamente comum, o conhecimento acerca dessa neoplasia benigna é de fundamental importância ao cirurgião-dentista. Entre os recursos terapêuticos, a escleroterapia química tem sido empregada, apresentando resultados satisfatórios tanto clínicos quanto estéticos. Objetivo: apresentar as características clínicas do hemangioma, os métodos de diagnóstico e o tratamento realizado por meio de escleroterapia química. Relato de caso: são descritos dois casos clínicos de hemangiomas, diagnosticados em duas pacientes do sexo feminino, localizados um em lábio superior e outro em mucosa jugal, tratados por meio de injeção de solução esclerosante. Considerações finais: em ambos os casos, foi possível proporcionar a involução das lesões mediante procedimentos não cirúrgicos, favorecendo, no período pós-operatório, o conforto e a estética, demonstrando a eficiência e a segurança na utilização do oleato de monoetanolamina a 5\% (Ethamolin $\left.^{\circledast}\right)$.

Palavras-chave: Escleroterapia. Ethamolin ${ }^{\circledR}$. Hemangioma. Malformações vasculares. Tratamento.

\section{Introdução}

Atualmente, o hemangioma é descrito pela Organização Mundial de Saúde como sendo uma neoplasia vascular benigna, apresentando um estágio de rápido crescimento com proliferação anormal dos vasos sanguíneos e células endoteliais e podendo provocar danos desagradáveis ao paciente, seguida por uma involução gradual. ${ }^{1}$ De acordo com Neville et al. ${ }^{1}$ (2009), são os tumores mais comuns da infância, com maior incidência em indivíduos do sexo feminino, manifestando-se mais em brancos do que em outros grupos raciais. A localização mais acometida por essa patologia é a região de cabeça e pescoço, ocorrendo em cerca de $60 \%$ dos casos, e na cavidade oral afeta com maior frequência a região de mucosa jugal, lábios, língua e palato.

Segundo Dias et al. ${ }^{2}$ (2013), os hemangiomas tiveram conceito e classificação, enquanto patologia, modificados ao longo dos anos. Por muito tempo, não houve uma classificação diagnóstica aceita universalmente, dificultando a elaboração de condutas bem como as comparações entre os tratamentos executados. Depois de várias tentativas de torná-las mais adequadas, em 1982, com melhor entendimento da angiogênese, Gontijo et al. ${ }^{3}$ (2004) propuseram que as anomalias vasculares fossem divididas em duas categorias distintas: hemangiomas e malformações vasculares. Essas manifestações são diferenciadas com base em características celulares, aparência clínica e história natural.

São várias as formas terapêuticas relatadas na literatura para as lesões vasculares, que demandam ainda muitos estudos e discussões. Não há um tratamento globalmente utilizado, mas aquele indicado especificamente para cada caso, respeitando as características da lesão e as necessidades particulares do paciente..$^{4-6}$ 
Tendo em vista que a queixa principal dos pacientes portadores de tal patologia é o distúrbio estético, a abordagem por meio da escleroterapia vem sendo utilizada com resultados clínicos satisfatórios em pequenas lesões, sem a necessidade de intervenção cirúrgica posterior, sendo uma opção conservadora, viável e de baixo custo. ${ }^{5} \mathrm{O}$ sucesso dessa terapia reduz a morbidade e os custos do tratamento cirúrgico, evitando também algumas complicações, como cicatriz, parestesia e/ou hemorragias, dependendo do tamanho e da localização da lesão. ${ }^{7}$

$\mathrm{O}$ hemangioma se desenvolve com certa frequência nas áreas de cabeça e pescoço, e, embora tenha comportamento benigno, qualquer procedimento exercido sem a devida cautela pode desencadear um quadro de urgência ou emergência, como, por exemplo, uma hemorragia de grande porte, podendo levar o paciente a óbito. ${ }^{8}$

Por conta da importância clínica, por ter altas taxas de incidências e estar comumente associado a áreas de atuação do cirurgião-dentista, o conhecimento acerca dessa malformação ou neoplasia benigna, assim como de seus riscos e formas de tratamento, possui uma grande relevância científica.

O objetivo do presente trabalho é relatar o tratamento de dois casos clínicos de hemangioma, localizados em lábio e mucosa jugal, por meio da escleroterapia química, com utilização de solução de oleato de monoetanolamina (OM) a 5\% (nome comercial: Ethamolin), considerando as características clínicas e os métodos de diagnóstico da lesão, diferenciando-a de outras anomalias vasculares.

\section{Materiais e método}

Trata-se de um estudo descritivo observacional do tipo relato de caso, de dois pacientes com diagnóstico anatomopatológico de hemangioma na região bucomaxilofacial, sendo ambos do sexo feminino, atendidos no Centro de Especialidades Odontológicas do município de Crato.

A história relatada neste estudo foi obtida diretamente dos pacientes por meio de informações verbais bem como de dados dos resultados de exames diagnósticos. Para análise dos resultados, após o tratamento, os pacientes foram reavaliados nas consultas de retorno, realizadas no mesmo local da intervenção.

A concordância com o termo de compromisso do investigador e com a declaração de confidencialidade dos dados do sujeito de pesquisa assegura o sigilo dos dados coletados e a utilização de tais informações unica e exclusivamente para fins científicos, preservando, integralmente, o anonimato dos pacientes.

Um termo de consentimento livre e esclarecido, seguindo todas as exigências do Comitê de Ética em Pesquisa do Centro Universitário Doutor Leão Sampaio, foi emitido em duas vias, ambas assinadas pelo paciente e pelo pesquisador, assegurando a preservação da identidade e o direito daquele de se desvincular da pesquisa a qualquer momento.
A revisão de literatura se deu a partir de buscas bibliográficas em bases de dados, como SciELO, Lilacs, Bireme e BVS, utilizando os descritores hemangioma, hemangioma capilar e escleroterapia; foram incluídos artigos escritos em português e publicados entre 1998 e 2017.

\section{Relato dos casos}

\section{Caso I}

Paciente M. E. A. F., normosistêmico, gênero feminino, estudante, 11 anos de idade, compareceu ao Centro de Especialidades Odontológicas Regional, em Crato, após encaminhamento de uma unidade básica de saúde do mesmo município, para avaliação e possível realização de biópsia em lesão acometendo lábio superior.

Durante anamnese inicial, paciente e sua responsável (mãe) relataram que a lesão surgiu meses após o nascimento, e outros profissionais da área já haviam avaliado há alguns anos, diagnosticado e orientado a não realização de intervenção, pois a lesão iria regredir com o avanço da idade. Entretanto, não houve mudança no quadro.

A avaliação clínica evidenciou lesão localizada em região anterior (linha média) do lábio superior (Figura 1). A lesão apresentava aumento de volume de coloração anormal (vermelho-arroxeada), nódulo séssil, superfície lisa e irregular, de consistência amolecida, que se aproximava do tecido normal do lábio, causando projeção anteroposterior, desconforto estético e constante trauma com abundante sangramento envolvido (segundo relatos) (Figura 2). A paciente queixava-se de dor na região à palpação. Ainda durante a avaliação clínica inicial, foi realizada a manobra de vitropressão (compressão feita com uma lâmina de vidro), e observado que a lesão mudava de coloração significativamente (provável isquemia).

Figura 1 - Aspecto clínico inicial do caso l: vista frontal

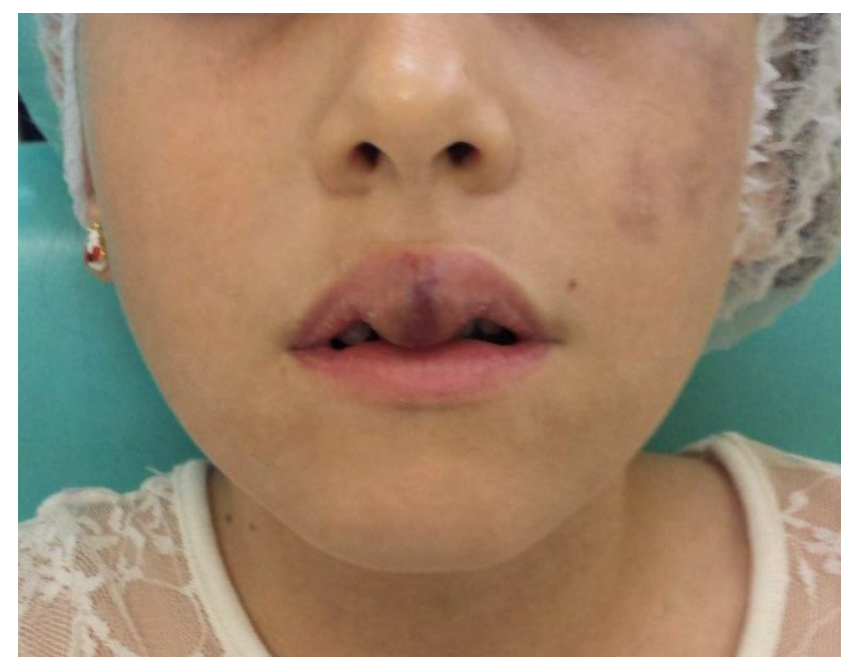

Fonte: autores. 
Figura 2 - Aspecto clínico inicial do caso I: lesão nodular vermelho-arroxeada

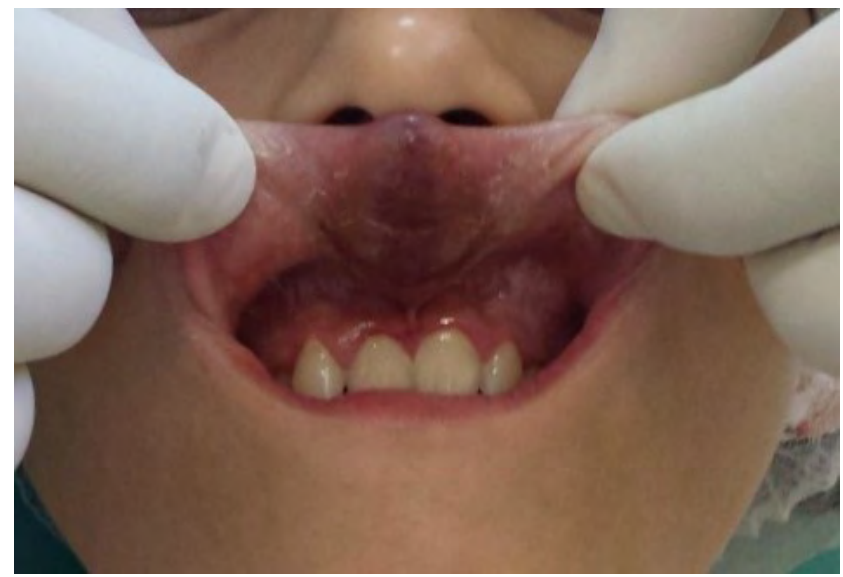

Fonte: autores.

Foi realizado ainda exame radiográfico periapical em região anterior, para descartar possível alteração dentária e/ou envolvimento ósseo. A conclusão do diagnóstico clínico foi hemangioma infantil acometendo lábio superior. Os familiares foram orientados a respeito da patologia e informados sobre as possíveis formas de tratamento, optando pela escleroterapia química intralesional com o uso de $\mathrm{OM}$ a $5 \%$.

$\mathrm{O}$ tratamento foi realizado sob anestesia terminal infiltrava na área, visto que se tratava de uma criança e a aplicação da medicação causa um certo desconforto. A substância (Ethamolin ${ }^{\circledR}$, uso adulto, injetável intravenosa, Zest) foi diluída em água para injeção na proporção de 1:2, resultando em uma concentração de $1,66 \%$.

Por se tratar de uma lesão não muito extensa, foi utilizado $1,8 \mathrm{ml}$ por aplicação, divididos em três pontos distintos. As doses foram aplicadas com o uso de uma seringa carpule, utilizando o próprio tubete anestésico esvaziado para anestesia do paciente e uma agulha gengival curta (Unoject $30 \mathrm{~g}$; $25 / 03 \mathrm{~mm}$ ). Foram realizadas quatro aplicações com intervalo de sete dias e uma última aplicação após 15 dias, totalizando cinco aplicações.

Com a área anestesiada, a paciente pouco referiu dor ou desconforto durante e logo após as sessões. A responsável relatou que, nos dias das aplicações, a filha referia ardor na área e um desconforto aliviado com o uso de analgésicos prescritos (dipirona sódica $200 \mathrm{mg} / \mathrm{ml}, 30$ gotas, de 6 em 6 horas) e compressa gelada.

Após duas sessões, tornou-se nítida a melhora do quadro, e, com o término do tratamento, pôde-se observar uma diminuição significativa da lesão (Figuras 3 e 4), cessando os traumas e o desconforto na área. $\mathrm{O}$ resultado apresentou-se esteticamente aceitável, e constatou-se a satisfação da paciente que, inclusive, se demonstrava mais vaidosa e extrovertida.
Figura 3 - Aspecto clínico final do caso l após 5 aplicações de oleato de monoetanolamina

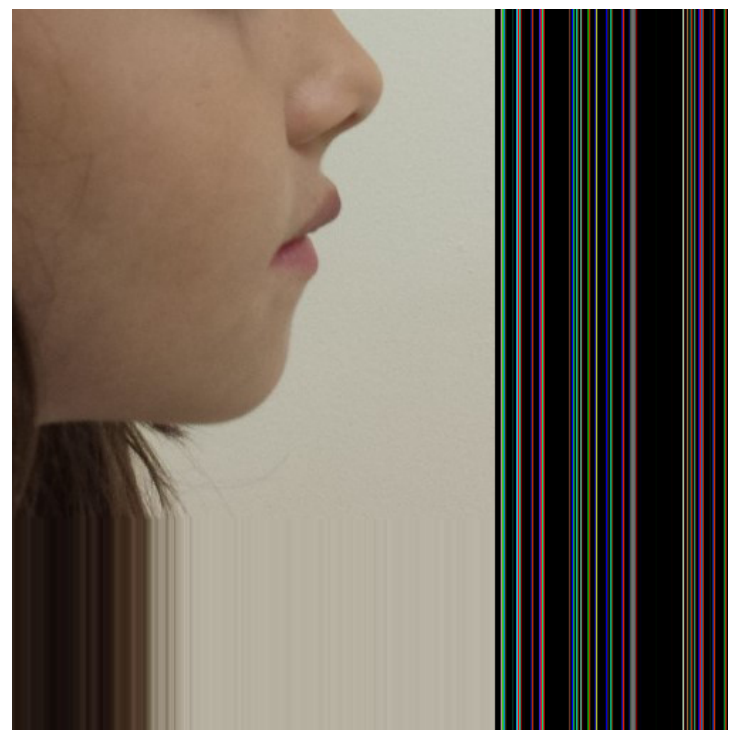

Fonte: autores.

Figura 4 - Aspecto clínico final do caso I após 5 aplicações de oleato de monoetanolamina (perfil)

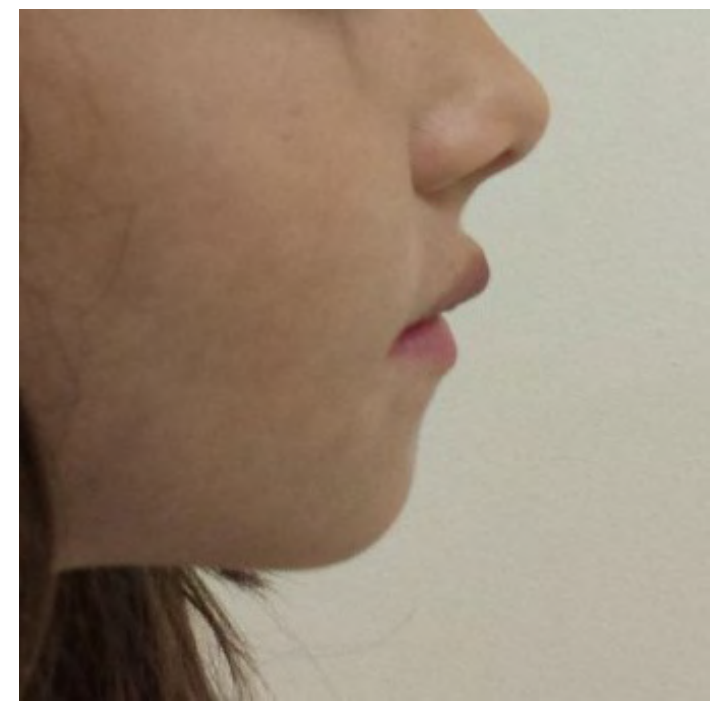

Fonte: autores.

\section{Caso II}

Paciente J. F. S., normosistêmico, gênero feminino, dona de casa, 27 anos de idade, compareceu ao Centro de Especialidades Odontológicas Regional de Crato, após encaminhamento de uma unidade básica de saúde do município de Várzea Alegre, CE, para avaliação e possível realização de biópsia em lesão acometendo mucosa jugal direita.

Durante a anamnese, a paciente não soube relatar ao certo o tempo de evolução da lesão, mas afirmou que há bastante tempo a observavara, e que outros dentistas já haviam realizado tratamento dentário, entretanto, nunca a informaram sobre o que poderia ser; e demonstrou-se muito preocupada com a possibilidade de ser uma alteração maligna. 
A lesão estava localizada na região da mucosa jugal do lado direito. Apresentava uma mácula azulada, superfície lisa e regular e, segundo relatos, causava constante trauma, com muito sangramento envolvido (Figura 5). A paciente queixava-se de dor na região à palpação, principalmente quando encontrava-se inflamada. Durante a avaliação clínica inicial, foi realizada a manobra de vitropressão, e observado que a lesão mudava de coloração significativamente (provável isquemia).

Figura 5 - Aspecto clínico inicial do caso Il: mácula azulada em mucosa jugal direita

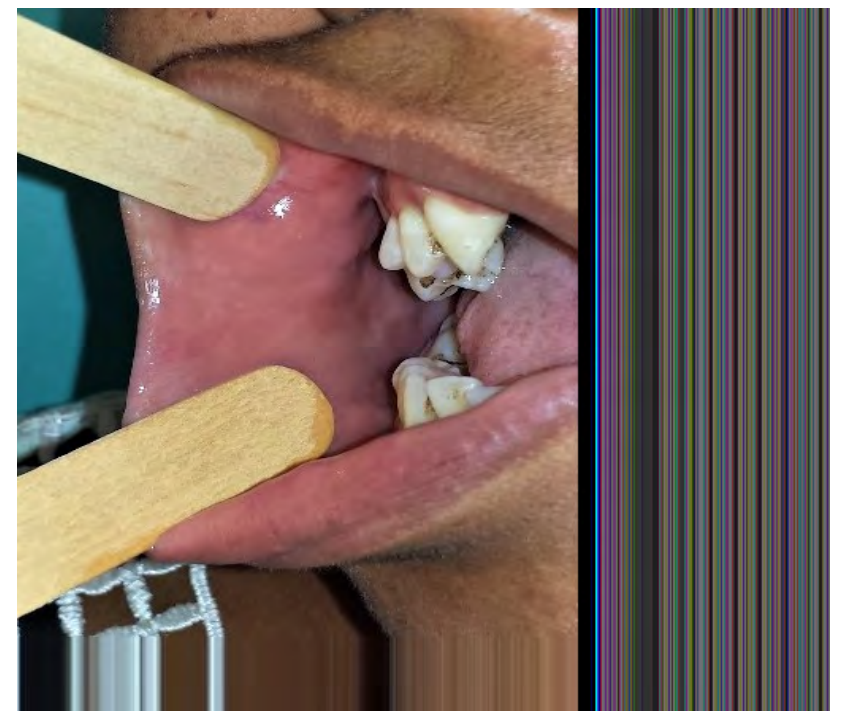

Fonte: autores.

A conclusão do diagnóstico clínico foi hemangioma. A paciente foi orientada acerca da patologia e, após ser informada sobre as possíveis formas de tratamento, optou-se, em consonância com o cirurgião-dentista, pelo tratamento com escleroterapia química intralesional com o uso de OM a $5 \%$.

O tratamento foi realizado sem a utilização de agente anestésico. A substância (Ethamolin ${ }^{\circledR}$, uso adulto, injetável intravenosa, Zest) foi diluída em água para injeção na proporção de 1:2, resultando em uma concentração de $1,66 \%$. A dose recomendada é de $1 \mathrm{ml}$ para cada $2 \mathrm{~cm}^{2}$ de lesão. Por se tratar de uma lesão não muito extensa, foi utilizado $1,8 \mathrm{ml}$ por aplicação, divididos em quatro pontos. As doses foram aplicadas com o uso de uma seringa carpule, utilizando o tubete anestésico esvaziado para a finalidade e uma agulha gengival curta (Unoject 30 g; 25/03 mm).

Foram realizadas três aplicações com intervalos de sete dias. Três dias após a terceira sessão, a paciente retornou ao Centro de Especialidades Odontológicas Regional, pois notou "algo estranho" e sintomatologia dolorosa à palpação. Ela foi avaliada, e observou-se necrose tecidual periférica. Quando indagada sobre possível quadro febril, a paciente negou. Foram prescritos colutório à base de gluconato de clorexidina $0,12 \%$ (duas vezes ao dia) e analgésicos para controle da queixa álgica. A paciente retornou após 15 dias, e puderam-se observar melhora do quadro infeccioso e total regressão da lesão (Figura 6).

Figura 6 - Aspecto clínico final do caso II: regressão total da lesão

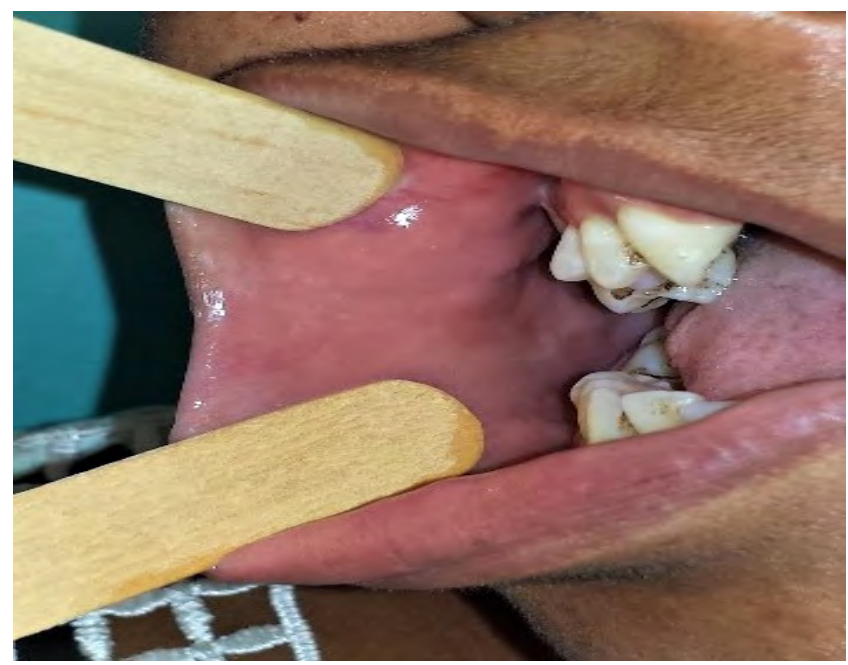

Fonte: autores.

\section{Discussão}

Andrade et al..$^{9}(2014)$ referem-se ao hemangioma como sendo um tumor do endotélio vascular, possuindo comportamento benigno, sendo caracterizado por um estágio inicial de intensa proliferação, seguido, na maioria dos casos, por involução espontânea. Tem grande incidência nas áreas de cabeça e pescoço e pode ser encontrado na cavidade oral, causando ulceração, dor, sangramento, infecção secundária e deformação do tecido. ${ }^{10}$

Nos dois casos apresentados, o gênero e a localização anatômica coincidem com os estudos de prevalência de Neville et al. ${ }^{1}$ (2009), em que os autores afirmam que o hemangioma acomete mais mulheres do que homens, surgindo na região de cabeça e pescoço em $60 \%$ dos casos, e na boca, principalmente em língua, mucosa jugal, palato e lábios.

$\mathrm{O}$ primeiro caso apresentado concorda ainda com Neville et al. ${ }^{1}$ (2009) em relação à idade, tendo sido relatado tumor na infância, conforme a responsável pela paciente (mãe); corrobora também com a análise feita por Seo et al. ${ }^{5}$ (2009), em que os autores declaram que grande parte das lesões não pode ser reconhecida no nascimento, entretanto, tende a surgir durante os dois primeiros meses.

Histologicamente, no hemangioma, diferentemente das malformações vasculares, há uma proliferação das células endoteliais, resultante do aumento da atividade mitótica, formando um acúmulo de capilares com parede delgada, delimitados por um estroma escasso de tecido conjuntivo. ${ }^{11,12} \mathrm{O}$ hemangioma comumente é caracterizado por três fases distintas: proliferação, involução e retorno à condição normal. A primeira fase equivale ao período de intensa proliferação e rápido crescimento, 
ocorrendo entre o nascimento e o primeiro ano de idade, em que é possível observar um aumento da atividade celular do endotélio que pode ou não vir a desenvolver um lúmen vascular. A fase de involução refere-se ao período de regressão, compreendendo, normalmente, de um a sete anos de idade. $\mathrm{E}$ a fase de retorno à condição normal sucede a completa regressão da lesão, sendo caracterizada pela presença de inúmeros capilares, alimentando esses vasos e drenando veias enfileiradas por um endotélio maduro e plano. ${ }^{5}$

Clinicamente, ambas as lesões relatadas compartilham dos mesmos aspectos referidos por Mandú et al. ${ }^{6}$ (2013), apresentando-se como uma mácula ou um nódulo arroxeado, cuja coloração pode variar de vermelho intenso a roxo, de acordo com localização, profundidade e grau de congestão do tecido. Em geral, essas lesões são relativamente flácidas à palpação, com limites difusos ou circunscritos e com superfície nodular ou lisa. As pacientes referiram ainda episódios de sangramento relacionados ao trauma e dor à palpação, quando as lesões se encontravam inflamadas.

O diagnóstico diferencial entre os hemangiomas e outros tumores ou malformações vasculares é determinado de forma simples e segura por meio de anamnese, exame clínico e manobras semiotécnicas. Tendo em vista que a biópsia é contraindicada, devido ao risco de hemorragias, foi utilizada a diascopia, ou vitropressão, em ambos os casos relatados, constituindo-se um importante auxiliar na conclusão do diagnóstico, pois a compressão aplicada sobre a lâmina de vidro fez com que a lesão assumisse momentaneamente uma coloração pálida, reduzisse de tamanho em função do esvaziamento vascular e, finalmente, após a remoção, regressasse ao volume inicial, podendo-se, assim, estabelecer o diagnóstico clínico sugestivo, descartando hipóteses de quaisquer lesões pigmentadas. ${ }^{11}$ Outra forma de se obter o diagnóstico é por meio da punção aspirativa ou do uso de exames de imagem, como angiografia, que, por meio da injeção de um produto de contraste, facilita a observação completa dos vasos sanguíneos.

A telangiectasia é caracterizada pela dilatação de pequenos vasos sanguíneos e capilares de parede delgada, aparentes na superfície da pele e mucosa, ${ }^{13}$ que, juntamente com o sarcoma de Kaposi, mucoceles, cistos de retenção e granuloma piogênico, está entre as hipóteses que constituem o diagnóstico diferencial desse tipo de lesão. ${ }^{5}$ No caso do hemangioma intraósseo, por conta da sua característica radiológica, pode ser facilmente confundido com o osteosarcoma ou com cistos odontogênicos. ${ }^{4,11}$ O diagnóstico final, por sua vez, somente pode ser estabelecido pelo exame histopatológico.

Em relação ao tratamento, Boraks ${ }^{14}$ (2001) alega que se deve ponderar com relação a localização e extensão, podendo optar por intervir de maneira ativa ou conservadora, por meio de procedimentos cirúrgicos ou do emprego de medicamentos. Embora a proservação seja a conduta mais frequentemente estabelecida, devido a altas taxas de involução espontânea dessas lesões, ${ }^{5}$ Andrade et al. ${ }^{9}$ (2014) afirmam que, em casos como os apresentados neste artigo, em que a lesão evolui com complicações frequentes, quando localiza-se em áreas anatômicas em que a involução tende a ser mais lenta ou incompleta, como na região de parótida, em ponta nasal e nos lábios, ou são lesões de grandes dimensões, é indicado que seja realizado um tratamento ativo.

Para a escolha do tipo de tratamento, algumas características foram levadas em consideração, como tamanho, localização, duração e hemodinâmica da lesão, por meio da observação do fluxo sanguíneo, além da idade do paciente e da viabilidade da técnica a ser utilizada, optando, assim, pela escloroterapia química, que tem a vantagem de ser um procedimento menos invasivo e que não promove cicatriz aparente, apresentando poucas complicações. Entretanto, tem a desvantagem de não poder ser empregada em lesões muito extensas, pois o intenso fluxo sanguíneo e os espaços vasculares mais largos e difundidos acabam por diluir e proporcionar menor contato com as paredes dos vasos acometidos, resultando em um efeito mínimo ou inexistente do fármaco com o endotélio vascular. ${ }^{15}$

Esse recurso terapêutico é um dos mais utilizados. Para esse fim, inúmeras substâncias foram empregadas, como tetradecil sulfato de sódio, morruato de sódio, solução hipertônica associada a heparina e procaína (ou lidocaína), psiliato de sódio e OM. ${ }^{16,17}$ Nos dois casos apresentados, o agente esclerosante empregado foi o OM (Ethamolin®), utilizado principalmente para o tratamento de varizes no esôfago.

Essa substância química configura-se como a solução mais utilizada para esse fim, devido à menor chance de toxicidade. No hemangioma, age provocando uma irritação da camada íntima do endotélio vascular, consistindo em uma resposta inflamatória estéril, e consequentemente uma fibrose da parede do vaso, ocasionando posteriormente redução e possivelmente oclusão da luz vascular. ${ }^{17}$ Como referido nos dois casos tratados, essa substância, ao ser administrada, pode desencadear uma sensação de ardor logo após sua infiltração bem como formação de uma lesão ulcerada ou necrose da camada mais superficial, como visto no segundo caso clínico apresentado. No entanto, pretendendo reduzir ou eliminar tais desconfortos, podem-se lançar mão do uso de anestesia local previamente ou associada à aplicação, depositar a substância na porção mais profunda e central da lesão e realizar a prescrição de medicação analgésica. ${ }^{16}$

Embora essa seja uma técnica simples e aparentemente sem grandes complicações, atenções especiais devem ser tomadas durante a sua utilização. O emprego do OM é contraindicado em pacientes diabéticos não controlados e em áreas de infecção secundária. Há contraindicação também para gestantes, visto que pode desenvolver efeito nocivo ao 
feto. ${ }^{12,16}$ A injeção de um volume superior ao recomendado pode, além de induzir a necrose superficial, desencadear um quadro de reação anafilática em pacientes sensíveis a esse fármaco. ${ }^{5}$ Embora sejam raros os casos de choque anafilático decorrentes da utilização desse medicamento, o profissional precisa estar preparado para tratá-lo apropriadamente.

Em conformidade com o estudo de Sales et al. ${ }^{15}$ (2015), a dose e os intervalos da administração do Ethamolin devem ser estabelecidos de acordo com o tamanho da lesão, recomendando, de modo geral, que as aplicações sejam realizadas em sessões intercaladas com intervalo mínimo de sete dias entre elas, não excedendo o limite de $2 \mathrm{ml}$ por infiltração.

Os resultados do presente trabalho são coerentes com a literatura consultada, demonstrando que a escleroterapia é uma técnica efetiva e relativamente simples de ser realizada em ambiente ambulatorial, desde que executada com o máximo de cuidados, tendo efeitos colaterais discretos e passageiros nas áreas adjacentes à lesão.

\section{Considerações finais}

A injeção intralesional do agente esclerosante (OM a 5\%) utilizado em ambos os casos apresentados foi responsável por promover a involução parcial no primeiro caso e a involução total no segundo, de modo prático e seguro, favorecendo a recuperação estética e representando uma alternativa eficaz e pouco invasiva para o tratamento de hemangiomas orais. Cabe, porém, ressaltar que há a possibilidade de recidiva da lesão, e deve-se respeitar alguns critérios básicos, como avaliar as características clínicas do hemangioma, estabelecer o diagnóstico correto, ter conhecimento prévio da saúde sistêmica do paciente bem como das indicações e contraindicações da escleroterapia. Desse modo, a realização da referida técnica deve ser cercada de todos os cuidados necessários, como em qualquer procedimento cirúrgico.

\section{Abstract}

Hemangioma is a neoplasm of benign behavior, determined by abnormal proliferation of blood vessels. They are asymptomatic lesions, however, their progressive growth can cause local traumatic injuries, causing pain, ulcerations and even bleeding, which, depending on the location, becomes difficult to control, especially when of an arterial nature. With a considerable incidence in the head and neck region, in the oral cavity, it most frequently affects the region of jugal mucosa, lips and tongue. Due to its clinical relevance, because it is present in the area of practice of the dental surgeon and is relatively common, knowledge about this benign neoplasm is of fundamental importance. Among the therapeutic resources, chemical sclerotherapy is employed presenting satisfactory clinical and aesthetic results. Objective: the purpose of this study is to present the clinical characteristics of hemangioma, the diagnostic methods and the treatment performed through chemical sclerotherapy. Case report: this article describes the description of two clinical cases of hemangiomas diagnosed in two female patients, one located in the upper lip and the other in the jugal mucosa treated with sclerosing solution injection. Final considerations: in both cases, it was possible to provide the involution of the lesions by non-surgical procedures, favoring, in the postoperative period, comfort and aesthetics, demonstrating the efficiency and safety in the use of 5\% monoethanolamine oleate (Ethamolin ${ }^{\circledR}$ ).

Keywords: Sclerotherapy. Ethamolin ${ }^{\mathrm{TM}}$. Hemangioma. Vascular malformations. Treatment.

\section{Referências}

1. Neville BW, Damm DD, Allen CM. Bouquot JE. Patologia oral e Maxilofacial. 3. ed. Rio de Janeiro: Guanabara Koogan; 2009.

2. Dias GF, Franca LH, Fraiz FC, Wambier DS, Kozlowski Jr VA, Céspedes JM. Hemangioma bucal em crianças. UEPG Ci Saúde, Ponta Grossa 2013; 19(1):21-9.

3. Gontijo B, Pereira LB, Silva CMR. Malformações vasculares. An Bras Dermatol, Rio de Janeiro 2004; 79(1):7-25.

4. Cruz FLG, Carvalho RF, Carvalho MF, Sales LAR, Devito KL. Diagnóstico diferencial de hemangioma por meio da vitropressão. Rev Gaúcha de Odontologia, Porto Alegre 2011; 59(1):125-9.

5. Seo J, Utumi E, Zambon C, Pedron I, Rocha A. Escleroterapia de hemangioma labial. Rev Odontol, São Bernardo do Campo 2009; 17(34):106-12.

6. Mandú AL, Lira CR, Barbosa LM, Silva VC, Cardoso AJ. Escleroterapia de hemangioma: relato de caso. Rev Cir Traumatol Buco-Maxilo-fac, Camaragibe 2013; 13(1):71-6.

7. Ribas MO, Laranjeira J, Sousa MH. Hemangioma bucal: escleroterapia com oleato de etanolamina. Revisão da literatura e apresentação de caso. Rev Clín Pesq Odontol, Curitiba 2004; 1(2):31-6.

8. Assis GM, Silva SRP, Moraes PH, Amaral JIQ, Germano AR. Hemangioma de língua: relato de caso. Rev Cir Traumatol Buco-Maxilo-fac, Camaragibe 2009; 9(2):59-66.

9. Andrade RF, Silva FD, Vilela Jr RD, Dias MA, Oliveira LR. Abordagem cirúrgica em hemangioma cavernoso em língua relato de caso. Rev da Univ Vale do Rio Verde, Três Corações 2014; 12(1):504-12.

10. Jaeger F, Alvarenga RL, Galizes BF, Girardi GP, Alvarenga GL, Leal RM. Escleroterapia com oleato de etanolamina a $5 \%$ em hemangioma oral: relato de caso clínico. Rev Port Estomatol Médico Dent Cir Maxilofac, Lisboa 2013; 54(2):91-4.

11. Rocha LB, Pádua JM, Martins RH, Lia RCC. Hemangioma da cavidade bucal. Rev Gaúcha de Odontol 2000; 48(3):1502.

12. Zanettini I, Zanettini RM, Gollo G. Escleroterapia como alternativa de tratamento de lesões vasculares bucais. Rev Clín Pesq Odontol, Curitiba 2005; 2(2):119-26.

13. Guarnieri CL, Patuzzi E, Jardim JJ, Barbachan e Silva B. Manejo odontológico da Síndrome de Rendu-Osler-Weber: relato de caso. Rev da Associação Paulista de Cirurgiões-Dentistas 2016; 70(3):256-9.

14. Boraks S. Diagnóstico bucal. 3. ed. São Paulo: Artes médicas; 2001. 
15. Sales PH, Vasconcelos RB, Meneses AM, Santos DF. Utilização de agente esclerosante no tratamento de hemangioma lingual - relato de caso. Rev Odontol Universidade Cidade de São Paulo 2015; 27(3):253-7.

16. Toledo HJB, Castro EVFL, Castro AL, Soubhia AMP, Salvador Junior FB. Hemangioma cavernoso de lábio inferior: caso clínico. Rev Odontol de Araçatuba 2004; 25(1):09-11.

17. Wang L, Oliveira D, Consolaro A, Perez F. Tratamento de hemangioma bucal com agente esclerosante. ROBRAC 1998; $7(24): 2022$.

\section{Endereço para correspondência:}

Erick Alves Pereira

Rua Pe. Cícero Coutinho, 70, Limoeiro

63030-260, Juazeiro do Norte, CE, Brasil

Telefone: (88) 99913-6262

E-mail: erickalvesp1@hotmail.com

Recebido: 03/09/18. Aceito: 25/09/18. 